\title{
Skewed non-Fermi liquids and the Seebeck effect
}

\author{
Antoine Georges $\oplus^{1,2,3,4}$ and Jernej Mravlje $\oplus^{5}$ \\ ${ }^{1}$ Collège de France, 11 place Marcelin Berthelot, 75005 Paris, France \\ ${ }^{2}$ Center for Computational Quantum Physics, Flatiron Institute, 162 5th Avenue, New York, New York 10010, USA \\ ${ }^{3}$ CPHT, CNRS, Ecole Polytechnique, IP Paris, F-91128 Palaiseau, France \\ ${ }^{4}$ DQMP, Université de Genève, 24 quai Ernest Ansermet, CH-1211 Genève, Switzerland \\ ${ }^{5}$ Department of Theoretical Physics, Institute Jožef Stefan, Jamova 39, SI-1001 Ljubljana, Slovenia
}

(Received 25 February 2021; revised 25 October 2021; accepted 27 October 2021; published 22 November 2021)

\begin{abstract}
We consider non-Fermi liquids in which the inelastic scattering rate has an intrinsic particle-hole asymmetry and obeys $\omega / T$ scaling. We show that, in contrast to Fermi liquids, this asymmetry influences the low-temperature behavior of the thermopower even when the impurity scattering dominates. Implications for the unconventional sign and temperature dependence of the thermopower in cuprates in the strange metal (Planckian) regime are emphasized.
\end{abstract}

DOI: 10.1103/PhysRevResearch.3.043132

\section{INTRODUCTION}

Besides its relevance to thermoelectricity, the Seebeck effect provides invaluable insights into the fundamental physics of materials [1-3]. The Seebeck coefficient $S$ (thermopower) is sensitive to the balance between holelike and electronlike excitations. It is negative when electrons dominate, positive when holes dominate, and vanishes when particle-hole symmetry holds. In many cases, the particle-hole asymmetry originates in the band structure of the material: It is controlled by the number (density of states) and velocities of the two types of excitations. However, it has been recognized that another source of asymmetry may also influence the Seebeck coefficient: that of the lifetime (scattering rate) of these excitations. Although this has been discussed theoretically [4-12] and put forward as a possible mechanism for materials in which the sign of $S$ is found to be opposite to that predicted by band structure $[4,11,13,14]$, it has received comparatively less attention. One of the reasons is that, as detailed below, the particle-hole asymmetry of the inelastic scattering rate does not influence $S$ at low temperature for metals obeying Fermi-liquid theory when impurity scattering is also present.

In this paper, we show that the situation is entirely different in correlated metals which do not obey Fermi-liquid theory. We consider a family of non-Fermi liquids in which the inelastic (electron-electron) scattering rate $\Gamma_{\text {in }}$ obeys $\omega / T$ scaling (with $\omega$ being the energy of an excitation counted from the Fermi level). We demonstrate that in "skewed" nonFermi liquids where the scaling function has an odd-frequency component, this particle-hole asymmetry affects the low- $T$

Published by the American Physical Society under the terms of the Creative Commons Attribution 4.0 International license. Further distribution of this work must maintain attribution to the author $(s)$ and the published article's title, journal citation, and DOI. behavior of the Seebeck coefficient down to $T=0$, even in the presence of impurity scattering. This is an unexpected finding because the impurity scattering is temperature independent, whereas the electron-electron scattering diminishes upon cooling down and vanishes at $T=0$. The sign of $S$ can be reversed in comparison to that expected from the band structure. The case of a "Planckian" metal [15-21] with $\Gamma_{\text {in }} \propto \omega, T$ turns out to be particularly interesting. In that case, $S / T$ ultimately diverges logarithmically at low temperature. However, the temperature dependence and sign of $S / T$ over an extended temperature range are strongly affected by the particle-hole asymmetry of $\Gamma_{\text {in }}$. As discussed below, this may be relevant to the understanding of the Seebeck coefficient of cuprate superconductors, especially close to the critical doping where the pseudogap opens and a logarithmic dependence of the specific heat is observed [22].

The paper is organized as follows: In Sec. II we describe the Kubo-Boltzmann formalism used in our calculations of the Seebeck coefficients, taking explicit account of the skewness (particle-hole asymmetry) of the inelastic scattering rate. In Sec. III we describe our main results that reveal the unusual effect of skewness in non-Fermi liquids and contrast it to the more moderate behavior in Fermi liquids. In Sec. IV we discuss the implication of our findings for experiments. Technical details are delegated to the Appendices: In Appendix A we give a derivation of the transport equations starting from the Kubo formalism, in Appendix B we discuss the $\omega / T$ scaling properties of the considered class of non-Fermi liquids, and in Appendix $\mathrm{C}$ we elaborate on the behavior of the Seebeck coefficient at higher temperatures.

\section{SEEBECK COEFFICIENT AND PARTICLE-HOLE ASYMMETRIC INELASTIC SCATTERING}

We recall that in metals, for noninteracting electrons and in the presence of elastic scattering only, the Seebeck coefficient 
at low temperature $T$ is given by

$$
\frac{S}{T}=-\frac{k_{B}}{e} \frac{\pi^{2}}{3} \frac{\Phi_{0}^{\prime}}{\Phi_{0}} .
$$

This expression involves the transport function $\Phi(\varepsilon)=$ $2 \int d^{d} k /(2 \pi)^{d} v_{\mathbf{k}}^{2} \delta\left(\varepsilon-\varepsilon_{\mathbf{k}}\right)$ at the Fermi level $\Phi_{0}=\Phi\left(\varepsilon_{F}\right)$ and its derivative with respect to energy $\Phi_{0}^{\prime}=\Phi^{\prime}\left(\varepsilon_{F}\right) \cdot v_{\mathbf{k}}=$ $\left(\nabla_{\mathbf{k}} \varepsilon_{\mathbf{k}}\right)_{\alpha} / \hbar$ denotes the electron velocity in the direction $\alpha=$ $x, y, z$ considered (we set $\hbar=k_{B}=1$ in most of the following). In this simplest description, $S / T$ does not depend on the magnitude of the scattering rate, and its sign is determined by the particle-hole asymmetry of the band structure encoded in the transport function: $S / T>0$ for a holelike band structure $\left(\Phi_{0}^{\prime}<0\right)$ and $S / T<0$ for an electronlike band structure $\left(\Phi_{0}^{\prime}>0\right)$.

We now consider the effect of both elastic and inelastic scattering (that is, the impurity and the electron-electron scattering, respectively), writing the total scattering rate as

$$
\Gamma(T, \omega)=\gamma+\Gamma_{\text {in }}(T, \omega) .
$$

It is convenient for our purpose to decompose the scattering time into components which are even and odd in frequency:

$$
\tau_{ \pm}(T, \omega)=\frac{1}{2}\left[\frac{1}{\Gamma(T, \omega)} \pm \frac{1}{\Gamma(T,-\omega)}\right] .
$$

Note that we assume that the elastic scattering rate $\gamma$ is isotropic and that the inelastic scattering rate only depends on frequency and not on momentum (i.e., it does not vary along the Fermi surface). The isotropy assumption allows us to keep the discussion simple and is sufficient to reveal the main effects that we wish to emphasize. Likewise, in our calculation of transport we do not take into account vertex corrections. This simplification is exact in models in which both the self-energy $\Sigma$ and the many-particle vertex are local (momentum independent) [23]. As discussed, e.g., in Ref. [24], inelastic electron-electron processes contributing to the conductivity in such models are entirely due to umklapp scattering. For recent discussions of the role of vertex corrections in transport, see Refs. [25-29]. In the following, we thus take $\Gamma_{\text {in }}(T, \omega)=-2 \operatorname{Im} \Sigma\left(\omega+i 0^{+}, T\right)$ (also neglecting possible distinctions between current and energy relaxation rates [30,31]).

Starting from the Kubo formula, one can derive the following expression for the Seebeck coefficient in the lowtemperature regime [32], as detailed in Appendix A:

$$
S=-\frac{k_{B}}{e} \frac{I_{1}(T)}{I_{0}(T)}
$$

in which

$$
\begin{gathered}
I_{1}(T)=\frac{T}{Z(T)} \frac{\Phi_{0}^{\prime}}{\Phi_{0}}\left\langle x^{2} \tau_{+}\right\rangle+\left\langle x \tau_{-}\right\rangle, \\
I_{0}(T)=\left\langle\tau_{+}\right\rangle+\frac{T}{Z(T)} \frac{\Phi_{0}^{\prime}}{\Phi_{0}}\left\langle x \tau_{-}\right\rangle .
\end{gathered}
$$

In these expressions, the frequency dependence of the scattering rates is expressed in terms of the scaling variable $x=\omega / T: \tau_{ \pm}=\tau_{ \pm}(T, x T)$, and we use the notation $\langle F(x)\rangle \equiv$ $\int_{-\infty}^{+\infty} d x F(x) / 4 \cosh ^{2} \frac{x}{2} . Z$ denotes the effective mass renormalization, which for a local theory is related to the real part of the self-energy by $1 / Z(T, \omega)=1+[\operatorname{Re} \Sigma(0)-\operatorname{Re} \Sigma(\omega)] / \omega$. Strictly speaking, the frequency dependence of $Z$ has to be kept in the integrals entering Eqs. (5) and (6). As we discuss in Appendix A, neglecting this effect is actually a good approximation, and we use it here to simplify the discussion. In a local Fermi liquid, $Z(T)$ coincides with the quasiparticle spectral weight and reaches a finite value at $T=0$, while in a non-Fermi liquid $Z(T)$ may vanish as $T \rightarrow 0$ [33]. In the absence of interactions $(Z=1)$ and for elastic scattering only ( $\tau_{+}=1 / \gamma, \tau_{-}=0$ ) we recover from Eqs. (4)-(6) the simple expression of Eq. (2) $\left(\langle 1\rangle=1,\left\langle x^{2}\right\rangle=\pi^{2} / 3\right)$. The effect of a possible temperature dependence of $Z(T)$ on both the Seebeck coefficient and the specific heat or entropy, for example, near a quantum critical point, has been previously discussed in the literature; see, e.g., Ref. [34]. In this paper, we focus on the effect of a particle-hole asymmetry of the scattering rate, i.e., on the terms involving $\left\langle x \tau_{-}\right\rangle$in Eqs. (5) and (6).

\section{FERMI LIQUIDS VERSUS NON-FERMI LIQUIDS}

\section{A. Fermi liquid}

We first consider a (local) Fermi liquid with an inelastic scattering rate:

$$
\Gamma_{\text {in }}(T, \omega)=\lambda\left[\omega^{2}+(\pi T)^{2}+a \omega^{3}+b \omega T^{2}\right]+\cdots .
$$

The key point is that the odd part of the scattering rate scales as $\omega^{3}, \omega T^{2} \sim x T^{3}$ and hence is subdominant as compared with the even-frequency part: The inelastic scattering rate of conventional Fermi liquids is asymptotically particle-hole symmetric at low energy. Adding the elastic scattering rate, we see that there are two regimes. In the "elastic regime," $\gamma$ dominates over the inelastic scattering rate: This holds for $\gamma \gtrsim$ $\lambda(\pi T)^{2}$ or alternatively for $T \lesssim T^{*}$ with $T^{*} \sim\left(\gamma / \pi^{2} \lambda\right)^{1 / 2}$ being a crossover temperature. In the "inelastic" regime $[\gamma \lesssim$ $\lambda(\pi T)^{2}$ or $T \gtrsim T^{*}$ ], inelastic scattering dominates [32]. Let us consider first the "inelastic" regime, in which $\tau_{+}$is of order $1 / T^{2}$ and $\tau_{-}$is of order $1 / T$. Hence, in the denominator $I_{0}$ of Eq. (4), $\left\langle\tau_{+}\right\rangle \sim 1 / T^{2}$ dominates over $T\left\langle x \tau_{-}\right\rangle \sim$ const. In contrast, in the numerator $I_{1}$, the odd term $\left\langle x \tau_{-}\right\rangle$has the same $1 / T$ temperature dependence as the even one $T\left\langle x^{2} \tau_{+}\right\rangle$. Taking into account that, in a Fermi liquid, the effective mass enhancement $1 / Z=m^{*} / m$ reaches a constant at low temperature, we obtain in the inelastic limit $T \gg T^{*}$

$$
\left.\frac{S}{T}\right|_{\text {in }} ^{\mathrm{FL}} \simeq-\frac{k_{B}}{e}\left[\left(12-\pi^{2}\right) \frac{1}{Z} \frac{\Phi_{0}^{\prime}}{\Phi_{0}}-\frac{12}{\pi^{4}}\left(c_{a} a+c_{b} b\right)\right],
$$

with coefficients $c_{a}=\left\langle x^{4} /\left(1+x^{2} / \pi^{2}\right)^{2}\right\rangle \simeq 6.51, \quad c_{b}=$ $\left\langle x^{2} /\left(1+x^{2} / \pi^{2}\right)^{2}\right\rangle \simeq 1.09$. Remarkably, the odd-frequency terms of $\Gamma_{\text {in }}$ directly contribute to $S$ in this limit, on equal footing with the band-structure term. This was, to our knowledge, first emphasized in Ref. [5], in which expression (8) was derived, and further discussed in Refs. [6-10,12]. We also note that the prefactor of the band-structure term is modified (from $\pi^{2} / 3 \simeq 3.29$ to $12-\pi^{2} \simeq 2.13$ ) as compared with the elastic (low- $T$ ) limit.

However, in the low- $T$ "elastic" limit $\left(\gamma \gg \lambda \pi^{2} T^{2}\right.$ or $T \ll$ $\left.T^{*}\right)$, this interesting effect disappears. Indeed, $\tau_{+} \sim 1 / \gamma$ is 


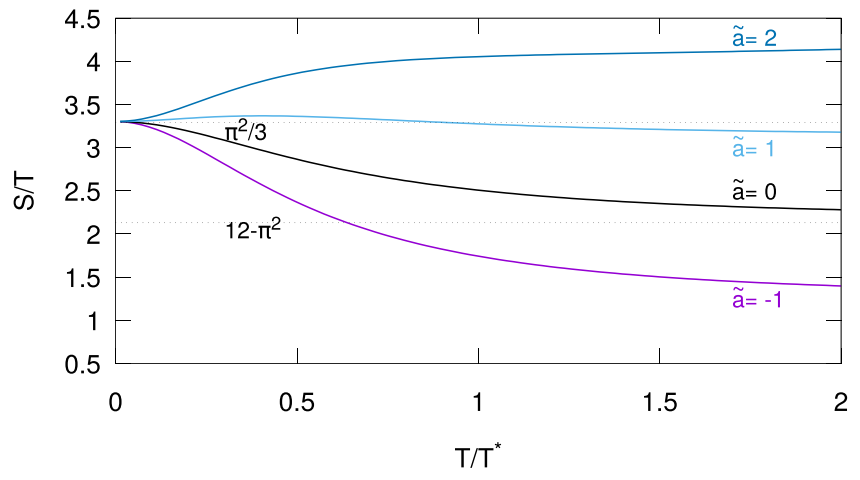

FIG. 1. Fermi liquid: $S / T$ in units of $\left|k_{B} / e \cdot \Phi_{0}^{\prime} / Z \Phi_{0}\right|$ vs $T / T^{*}$ for several values of the dimensionless particle-hole asymmetry parameters $\tilde{a} \equiv a Z \Phi_{0} / \Phi_{0}^{\prime}$ and taking $a=b$ (see text). This illustrates the crossover between the low- $T$ elastic-dominated regime which does not depend on asymmetry and the higher- $T$ inelastic-dominated one in which the asymmetry contributes. A holelike band contribution $\Phi_{0}^{\prime} / \Phi_{0}<0$ is considered here.

constant in this limit, while $\tau_{-}$vanishes as $T^{3}$, leading to

$$
\left.\frac{S}{T}\right|_{\mathrm{el}} ^{\mathrm{FL}} \simeq-\frac{k_{B}}{e} \frac{\pi^{2}}{3} \frac{1}{Z} \frac{\Phi_{0}^{\prime}}{\Phi_{0}} .
$$

Hence odd-frequency scattering does not contribute at low temperature. The conventional elastic value of Eq. (2) is recovered, with the notable difference that the prefactor is enhanced by the effective mass $1 / Z=m^{*} / m$. Indeed, it was emphasized in Ref. [35] that $S / T$ is proportional to the linear term in the specific heat $\left(\sim m^{*} / m\right)$ in many materials.

The crossover between the low- $T$ elastic limit and the high$T$ inelastic limit is illustrated in Fig. 1. The data in this plot as well as in Figs. 2 and 3 are obtained from evaluating the Seebeck coefficient with Eq. (4). In Appendix A, we show that the results are unchanged when evaluated from the full Kubo formula. We see that, in a Fermi liquid, the band value of the Seebeck coefficient enhanced by the effective mass effect $(Z)$ is recovered below the crossover temperature $T^{*}$. In that regime, the particle-hole asymmetry of the inelastic scattering has no influence on the Seebeck coefficient. In practice, $T^{*}$ can be estimated as the characteristic temperature at which the measured $T$-dependent contribution to the resistivity becomes comparable in magnitude to the residual resistivity at low $T$.

\section{B. "Skewed" non-Fermi liquids}

The washing out of the effects of the particle-hole asymmetry in the Fermi-liquid case happens because the odd-frequency terms in the inelastic scattering rate are subdominant in comparison to the even ones and hence do not contribute to $S$ at low $T$. We consider now a class of nonFermi liquids in which, in contrast, the odd-frequency terms are of the same order as the even-frequency ones, such that the inelastic scattering rate obeys a scaling form

$$
\Gamma_{\text {in }}(T, \omega)=\lambda(\pi T)^{v} g\left(\frac{\omega}{T}\right) .
$$

Here, $v$ is an exponent (we focus on $v \leqslant 1$ in the following), and the scaling function $g(x)$ contains both an even and
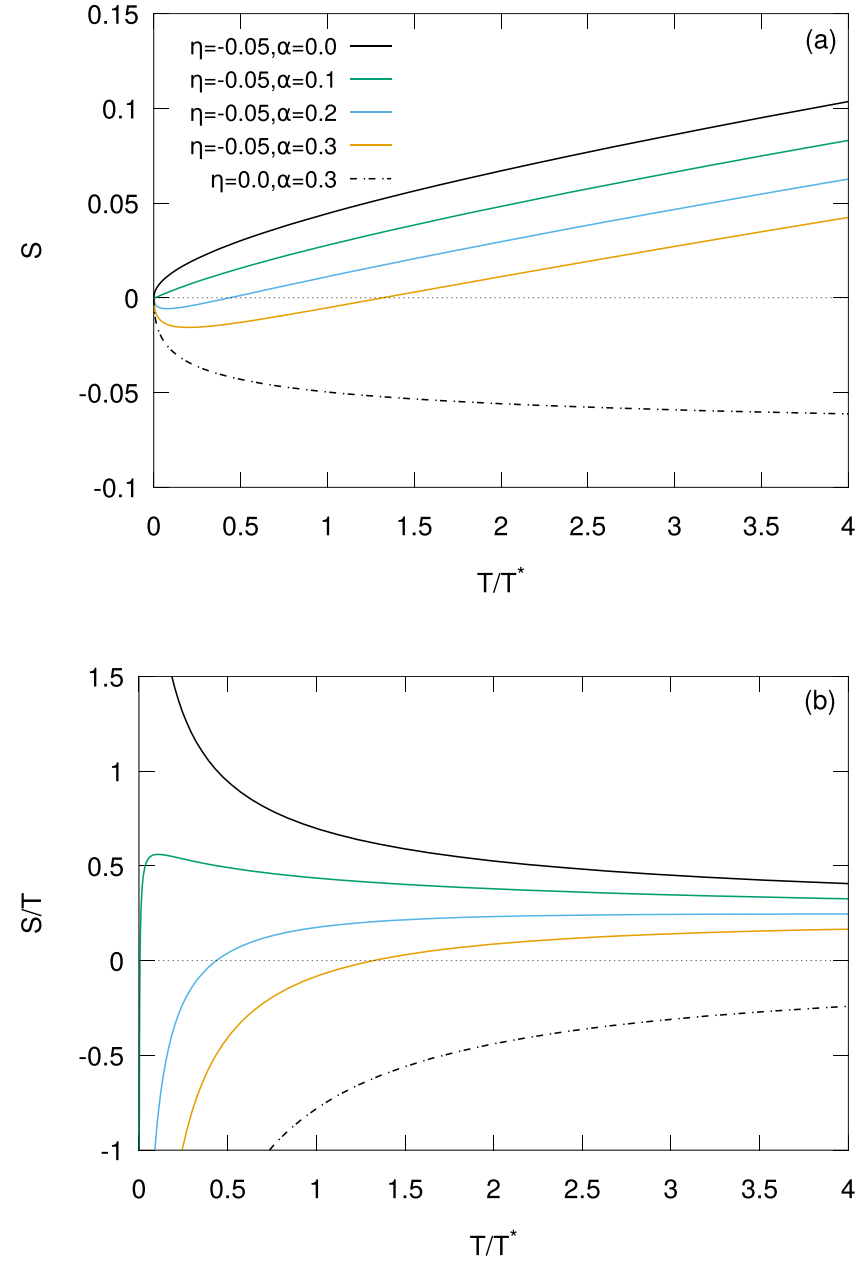

FIG. 2. "Skewed" non-Fermi liquid with $v=1 / 2$. (a) shows the Seebeck coefficient vs $T / T^{*}$ in units of $k_{B} / e$, for $\pi T^{*} / \gamma=0.2$ and several values of the asymmetry parameter $\alpha$. (b) shows $S / T$ in units of $k_{B} /(e \gamma)$. At low $T$, the sign of $S$ is seen to depend on $\alpha$, while at high $T$ all curves have the same sign, set by the band structure $\eta=\gamma \Phi_{0}^{\prime} / \Phi_{0}<0$ (holelike). The dash-dotted curve corresponds to the special case $\eta=0$ in which the sign of $S$ is determined solely by the scattering rate asymmetry (see text).

an odd component. It has a regular expansion at small $x$, so that for $\omega \lesssim T, \Gamma_{\text {in }} \sim \lambda(\pi T)^{\nu}\left[g(0)+g^{\prime}(0) T^{-1} \omega\right]+\cdots$, while $g(x) \sim c_{ \pm}^{\infty} \cdot|x|^{v}$ at large $x$. The effects discussed in this paper do not depend on the specific form of the scaling function $g(x)$. Systems obeying this scaling form with a noneven scaling function can be called "skewed non-Fermi liquids."

It is of course expected that systems such as doped Mott insulators should display a particle-hole asymmetry (see, e.g., Ref. [8]). However, while it is clear that such an asymmetry exists on energy scales comparable to electronic ones, it is a more demanding requirement that this asymmetry persists down to frequencies comparable to the temperature itself, as assumed in (10). Indeed, this does not apply in a Fermi liquid. In contrast, we note that the scaling form of Eq. (10) has been shown to apply in overscreened Kondo models controlled by a non-Fermi-liquid fixed point [36]. It is also relevant to the proximity of the quantum critical point of doped random-exchange Hubbard models, related to 


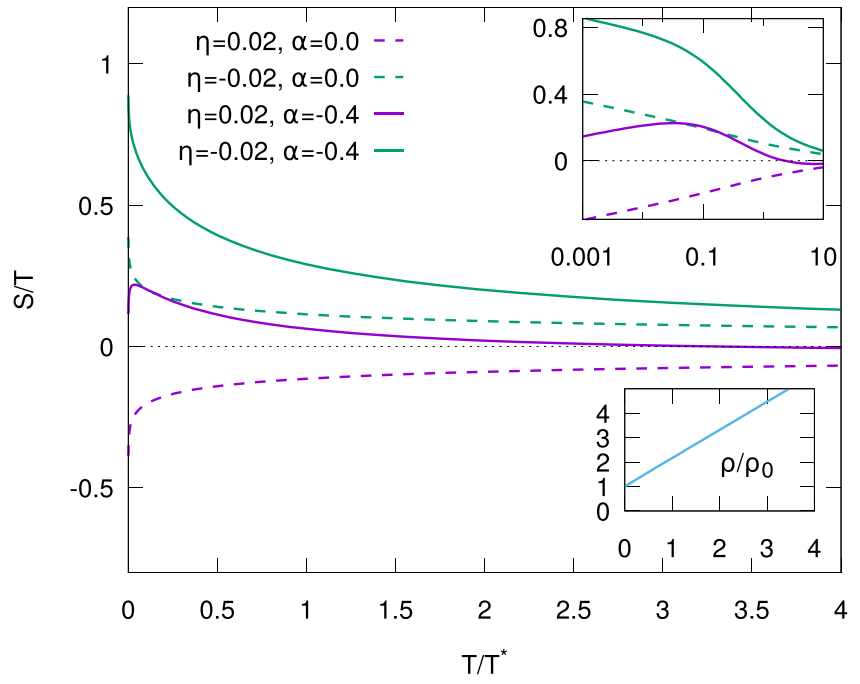

FIG. 3. Planckian metal. Temperature dependence of $S / T$ [in units of $\left.k_{B} /(e \gamma)\right]$ for two opposite values of the band-structure term $\eta=\gamma \Phi_{0}^{\prime} / \Phi_{0}$ : electronlike $\eta>0$ and holelike $\eta<0$. Solid (dashed) curves are for a particle-hole asymmetric (symmetric) scattering rate $[\alpha \neq 0(\alpha=0)] . \pi T^{*} / \gamma=1$. Top inset: $S / T$ on a logarithmic scale, emphasizing the low- $T$ behavior. Bottom inset: Linear dependence of the resistivity $\rho / \rho(T=0)=I_{0}(0) / I_{0}(T)$ on $T / T^{*}$.

Sachdev-Ye-Kitaev (SYK) models [37,38], as recently studied in Refs. [39-46].

For such models obeying conformal invariance at low energy, $g(x)$ is a universal scaling function that depends only on the exponent $v$ and on a "spectral asymmetry" parameter $\alpha$. Its exact form was derived in Ref. [36] and reads [with the normalization $g_{\alpha=0}(0)=1$ ]

$$
g(x)=\left|\Gamma\left[\frac{1+v}{2}+i \frac{x+\alpha}{2 \pi}\right]\right|^{2} \frac{\cosh (x / 2)}{\cosh (\alpha / 2) \Gamma[(1+v) / 2]^{2}},
$$

where $\Gamma(x)$ is the $\Gamma$ function. The $\omega / T$ scaling form is also relevant to the proximity of a quantum critical point associated with a strong-coupling fixed point [47].

The crossover temperature $T^{*}$ separating the "elastic" and "inelastic" limits now reads $\left(\pi T^{*}\right)^{v}=\gamma / \lambda$. In the low$T$ elastic limit, $\tau_{+} \sim 1 / \gamma$ and $\tau_{-} \sim-\lambda \gamma^{-2}(\pi T)^{v} g_{-}(x) \sim$ $-\gamma^{-1}\left(T / T^{*}\right)^{v} g_{-}(x)$. In this expression, $g_{-}(x)=[g(x)-$ $g(-x)] / 2$ is the odd-frequency component of the scaling function $g$. For the Seebeck coefficient at low $T$ one obtains

$$
\left.S\right|_{\mathrm{el}} ^{\mathrm{NFL}}=-\frac{k_{B}}{e}\left[\frac{\pi^{2}}{3} \frac{\Phi_{0}^{\prime}}{Z(T) \Phi_{0}} T-\frac{\lambda(\pi T)^{v}}{\gamma} c_{-}\right],
$$

in which $c_{-}=\left\langle x g_{-}(x)\right\rangle$ is a universal constant depending only on $g$. Ignoring at first the temperature dependence of the quasiparticle weight $Z(T)$, one sees that whenever $v \leqslant 1$, the particle-hole asymmetry of the inelastic scattering influences $\left.S\right|_{\mathrm{el}} ^{\mathrm{NFL}}$ even at the lowest temperatures, in sharp contrast to the Fermi-liquid case discussed above.

What is the influence of the temperature dependence of $Z$ ? For $v<1$ (we consider separately the $v=1$ case below), $Z$ vanishes at low $T$ as $\sim T^{1-v}$, and it can be shown (see Appendix B) that $T / Z(T) \sim \lambda(\pi T)^{v} / c_{Z}+T$ with $c_{Z}$ being a universal constant depending only on the scaling function $g$.
Hence we see that, remarkably, the two terms in the numerator $I_{1}$ of Eq. (4) have the same $T$ dependence: $T\left\langle x^{2} \tau_{+}\right\rangle / Z \sim T^{v}$ and $\left\langle x \tau_{-}\right\rangle \sim T^{\nu}$. One therefore obtains for $T \ll T^{*}$

$$
\begin{aligned}
\left.S\right|_{\mathrm{el}} ^{\mathrm{NFL}} & =-\frac{k_{B}}{e} \frac{\lambda}{\gamma}(\pi T)^{v}\left[\frac{\pi^{2}}{3 c_{Z}} \gamma \frac{\Phi_{0}^{\prime}}{\Phi_{0}}-c_{-}\right] \\
& =-\frac{k_{B}}{e}\left(\frac{T}{T^{*}}\right)^{v}\left[\frac{\pi^{2}}{3 c_{Z}} \eta-c_{-}\right] .
\end{aligned}
$$

The dimensionless parameter $\eta \equiv \gamma \Phi_{0}^{\prime} / \Phi_{0}$ is the ratio of the elastic scattering rate to the characteristic energy scale associated with the band-structure asymmetry. Hence, in this case, $S$ behaves as $T^{v}$ at low $T$, corresponding to a divergent slope $S / T$.

Expression (13) has several remarkable features. Firstly, we see that the odd-frequency inelastic scattering contributes to the low- $T$ Seebeck coefficient on equal footing with the even-frequency-elastic contribution. Both terms in Eq. (13) have the same $T$ dependence $\sim T^{v}$ but for different reasons: the first one because of the vanishing of $Z(T)$, and the second one because of the $T$ dependence of $\Gamma_{\text {in }}$. Secondly, in contrast to the former, this odd-frequency contribution is completely independent of the band-structure asymmetry: Its sign is dictated by that of the constant $c_{-}$, and thus by the intrinsic asymmetry of the inelastic rate scaling function. If this term dominates, the overall sign of the Seebeck coefficient can be opposite to that predicted by band-structure considerations in the low- $T$ limit, even when elastic scattering is present. This is one of the main results of this work. The odd-frequency contribution dominates over the first term when the dimensionless ratio $\eta=\gamma \Phi_{0}^{\prime} / \Phi_{0}$ is small, i.e., for clean-enough systems. Thirdly, we note that $S$ depends on both the inelastic constant $\lambda$ and the elastic rate $\gamma$. This is an unusual situation in which the strength of the scattering does not drop out of the value of $S$ at low $T$.

The behavior of Eq. (4) with temperature is investigated analytically in Appendix C. At high $T$, we obtain

$$
S \sim-\frac{k_{B}}{e}\left[\eta \frac{c_{2}}{c_{0}} \frac{T}{\gamma}+\eta \frac{c_{2}}{c_{0} c_{Z}}\left(\frac{T}{T^{*}}\right)^{\nu}+\frac{c_{1}}{c_{0}}\right],
$$

with $c_{n}=\left\langle x^{n} / g\right\rangle$. Hence, as long as $\eta=\gamma \Phi_{0}^{\prime} / \Phi_{0} \neq 0$, the behavior of $S$ at high temperature is dominated by the first term of Eq. (14) (with a linear dependence in $T$, the same sign as the band-structure result, but with a slope that is corrected by $c_{2} / c_{0}$ ). In contrast to the low- $T$ limit, the sign of the Seebeck coefficient is thus given by the band-structure term. Remarkably, in the absence of elastic scattering or band asymmetry $(\eta=0), S$ instead tends to a constant value $-c_{1} / c_{0} k_{B} / e$ which depends only on the universal scaling function and its asymmetry. A related finding was reported in Ref. [42] (see also Ref. [45]) in the context of SYK models, where $S$ was shown to be constant and determined by the spectral asymmetry $\alpha$ (related by holography to the electric field or charge of the black hole and to the ground-state entropy of these models).

In Figs. 2(a) and 2(b), we display the temperature dependence of $S$ and $S / T$, respectively, plotted vs $T / T^{*}$ in the non-Fermi-liquid case for several values of the asymmetry parameter $\alpha$ in Eq. (11). We see that at low $T$ the sign of $S$ can be changed by the scattering rate asymmetry, while it is 
set by the band-structure term at high $T$, except for vanishing $\eta$, where the Seebeck approaches a constant given by the last term of Eq. (14), instead.

The overall behavior at larger $T$ reveals a const $+T^{v}+T$ behavior at higher $T$ (with $v=1 / 2$ in Fig. 2), with a different coefficient of the $T^{v}$ term in the high- $T$ regime from that in the low- $T$ regime. We see that the larger the asymmetry, the larger the temperature at which the sign change occurs as compared with the symmetric case.

A remark is in order here. In microscopic models or materials realizations, the universal scaling form of the scattering rate (10) is only expected to apply below a certain cutoff, which is of the order of the bare electronic energy scales, e.g., the bandwidth. The physics discussed here obviously can only apply for temperatures below that cutoff. Hence, for our analysis of the $T$ dependence to be valid, the system should be clean enough that the crossover scale $T^{*}$ is smaller than such bare electronic energies at which nonuniversal effects will appear.

\section{Skewed Planckian metal}

We now discuss the case of a "Planckian" metal [15-21] $(v=1)$ with a particle-hole asymmetry scaling with $\omega / T$, so that $\Gamma_{\text {in }}=\pi \lambda g(0) T+\pi \lambda g^{\prime}(0) \omega+\cdots$ at low $\omega$ and $\omega<T$ ("skewed" Planckian metal). Setting $v=1$ in expression (13) would predict a linear dependence of $S$ at low $T$, with a slope that is given in terms of the mutual effect of the band-structure and inelastic scattering rate asymmetries. This behavior corresponds to a case where the quasiparticle weight is approximated by a constant. However, the quasiparticle weight actually vanishes logarithmically: $1 / Z(T) \sim 1+$ $\pi \lambda c_{Z}^{-1} \ln \Lambda / T$, with $\Lambda$ being a high-energy cutoff and $c_{Z}$ being a universal constant depending on the scaling function $g$; see Appendix B (note that $\lambda$ is dimensionless in the Planckian case). Hence, in the low- $T$ "elastic" limit $T \ll T^{*}=\gamma / \pi \lambda$,

$$
\left.\frac{S}{T}\right|_{\mathrm{el}} ^{\mathrm{SPM}} \sim-\frac{k_{B}}{e}\left[\pi \lambda \frac{\pi^{2}}{3 c_{Z}} \frac{\Phi_{0}^{\prime}}{\Phi_{0}} \ln \frac{\Lambda}{T}+\frac{\pi^{2}}{3} \frac{\Phi_{0}^{\prime}}{\Phi_{0}}-c_{-} \frac{\pi \lambda}{\gamma}+\cdots\right] .
$$

Thus, in a Planckian metal, $S / T$ ultimately diverges logarithmically at very low $T$. This corresponds to the logarithmic divergence of the effective mass (specific heat coefficient). The sign of the logarithmic term in $S$ is dictated by the band structure. In contrast, the last term in Eq. (15) is controlled by the odd-frequency part of the inelastic scattering and has a sign which can counteract the conventional band-structure effect corresponding to the second term in Eq. (15). Interestingly, we note that the "skewed" term dominates over the conventional one for cleaner systems $\eta / \lambda \lesssim 3 c_{-} / \pi$. The full temperature dependence is again given by Eq. (C5), setting $v=1$ and replacing $c_{Z}$ by $c_{Z} / \ln \Lambda / T$.

To illustrate these effects, we display in Fig. 3 the $T$ dependence of $S / T$ for a "skewed" Planckian metal. Two opposite signs of the band-structure term $\Phi_{0}^{\prime} / \Phi_{0}$ are considered. In the absence of a scattering rate asymmetry $(\alpha=0)$ it is seen that $S / T$ has a rather weak $T$ dependence (except at low $T$, where the logarithmic term becomes relevant), while it acquires significant $T$ dependence for $\alpha \neq 0$. Importantly, in the presence of an asymmetry, the sign of the Seebeck coefficient can be reversed in comparison to its band-structure value over a wide range of temperature.

\section{SUMMARY AND DISCUSSION}

In summary, our work reveals the importance of a particlehole asymmetry of the inelastic scattering rate in a class of non-Fermi liquids. This asymmetry influences the Seebeck coefficient even in the asymptotic low-temperature regime, when inelastic scattering events are comparatively rare as compared with elastic scattering on impurities.

A number of materials display non-Fermi-liquid behavior and an unconventional $T$ dependence of the Seebeck coefficient. This has been addressed in previous theoretical works, such as Refs. [34,48], which have emphasized the logarithmic divergence of $S / T$ in metals with a $T$-linear scattering rate. The $T$ dependence of $S$ close to a Fermi surface Lifshitz transition has been considered in Refs. [49,50]. However, to our knowledge, the key role of a particle-hole asymmetry of the inelastic scattering rate in non-Fermi-liquid metals with $\omega / T$ scaling has not been discussed before. The theory presented here may be relevant when both the temperature dependence and the sign of the Seebeck coefficient are found to be unconventional.

Cuprate superconductors in the "strange metal" regime display clear signatures of quantum criticality: $T$-linear resistivity (for reviews, see, e.g., Refs. [21,51,52]), a logarithmic divergence of the specific heat coefficient $C / T$ [22], and $\omega / T$ scaling observed in optical spectroscopy [53] and angular-resolved photoemission spectroscopy (ARPES) [54]. An increase of the in-plane $S / T$ at low $T$ reminiscent of Fig. 3 has been reported for $\mathrm{La}_{1.8-x} \mathrm{Eu}_{0.2} \mathrm{Sr}_{x} \mathrm{CuO}_{4}$ (EuLSCO) [55] at hole doping $p \simeq 0.21$ and $p \simeq 0.24$ and for $\mathrm{La}_{1.6-x} \mathrm{Nd}_{0.4} \mathrm{Sr}_{x} \mathrm{CuO}_{4}$ (Nd-LSCO) [56,57] at $p \simeq 0.24$, just above the critical doping $p^{*}$ at which the pseudogap phase terminates. Interestingly, $S$ was found to be positive at those doping levels, while simple considerations based on band structure and isotropic elastic scattering yield a negative value [58]. It is thus tempting to infer from these observations that the quantum critical (strange metal) regime of those cuprate superconductors may be described as a "skewed Planckian metal." We emphasize that experiments involving different controlled levels of disorder would play a decisive role in assessing the relevance of the mechanism proposed here, since the asymmetric term in Eq. (15) becomes larger for cleaner systems. For a discussion of the relevance of the present theory to the interpretation of recent measurements of the $a b$-plane and $c$-axis measurements of the Seebeck coefficient in Nd-LSCO, see Ref. [59].

In Eu-LSCO, Nd-LSCO, and also $\mathrm{Bi}_{2} \mathrm{Sr}_{2-x} \mathrm{La}_{x} \mathrm{CuO}_{6+\delta}$ (Bi2201) [60], the increase in $S / T$ at low $T$ observed experimentally appears consistent with a logarithmic dependence. Given the sign of the band-structure term for Nd-LSCO, one would expect from Eq. (15) a negative coefficient of this logarithmic term, in contrast to the experimental observation. This may suggest that the asymmetry term is actually dominant (as in Fig. 3) in the range of temperature of the measurement, in which the increase in $C / T$ is moderate [22].

As emphasized recently by Jin et al. [61], in the overdoped regime the Seebeck coefficient of the LSCO family 
remains positive $[62,63]$. These overdoped compounds behave as Fermi liquids at low enough temperature, however [64-66]. Hence, in view of the discussion above (Fig. 1), a particle-hole asymmetry of the inelastic scattering rate is unlikely to be responsible for this unexpected sign of $S$. It is possible that a particle-hole asymmetric dependence of the elastic scattering rate on momentum and energy is relevant to explain this observation $[51,67,68]$. We also note that the Seebeck coefficient of other single-layer cuprates such as $\mathrm{HgBa}_{2} \mathrm{CuO}_{4+\delta}$ (Hg1201) [69] and Bi2201 [70,71] has been reported to be negative in the overdoped regime.

Recently, the present theory was applied to experimental measurements of the $a b$-plane and $c$-axis Seebeck coefficients in Nd-LSCO [59]. The scattering rates used in this analysis were extracted from angular-dependent magnetoresistance measurements, which reveal that the elastic (temperature independent) rate is momentum dependent but that the inelastic one is not [20]. The theory was hence minimally extended as compared with the present work by using a momentum-dependent elastic lifetime and found to describe the experimental results well. We stress that the qualitative aspects (change of sign for the in-plane Seebeck coefficient due to inelastic scattering) are already well described by the simpler approach used here, which neglects the momentum dependence, but that quantitative aspects require the momentum dependence to be taken into account, especially for the out-of-plane Seebeck coefficient.

Our results are relevant also in the context of the nonFermi-liquid quantum critical point separating a metallic spinglass phase and a Fermi-liquid metal in doped SYK models, which has attracted a lot of attention recently $[43,44,46,72-$ 74]. Indeed, numerical results show strong spectral asymmetry in the quantum critical regime, possibly signaling a skewed non-Fermi liquid at the critical point [46]. We also note that in the context of transport in SYK models, the Seebeck coefficient has been emphasized as a probe of the universal ground-state entropy $[42,45]$.

Our work may also have relevance to twisted bilayer graphene and related systems, in which linear resistivity and Planckian behavior have been observed; see, e.g., Ref. [75]. A systematic investigation of thermoelectric effects and heat transport in these materials would be of great interest. We note that a recent study of twisted bilayer graphene reports a rich temperature dependence with changes of sign in the Planckian regime [76].

\section{ACKNOWLEDGMENTS}

We are grateful to Amir Ataei, Nicolas Doiron-Leyraud, Adrien Gourgout, Gaël Grissonnanche, Louis Taillefer, and Simon Verret for sharing their experimental data and for a collaboration and discussions on this topic. We also acknowledge discussions with Kamran Behnia, Christophe Berthod, Philipp Dumitrescu, Pablo Jarillo-Herrero, Olivier Parcollet, Subir Sachdev, Nils Wentzell, and Manuel Zingl. A.G. acknowledges support from the European Research Council (ERC-QMAC-319286). J.M. acknowledges funding by the Slovenian Research Agency (ARRS) under Programs No. P1-0044, J1-1696, and J1-2458. The Flatiron Institute is a division of the Simons Foundation.

\section{APPENDIX A: THERMOPOWER AND CONDUCTIVITY EVALUATED WITH THE KUBO FORMULA}

The conductivity $\sigma$, thermopower $S$, and (electronic) heat conductivity $\kappa$ are given by

$$
\sigma=e^{2} L_{11}, \quad S=-\frac{L_{12}}{e L_{11}}, \quad \kappa=T\left[L_{22}-\frac{L_{12}^{2}}{L_{11}}\right],
$$

in which $L$ are the Onsager coefficients and $e$ is the absolute magnitude of the electron charge. In the following, we set for simplicity $\hbar=k_{B}=e=1$ (except when restored in final results). Within the Kubo formalism, and neglecting vertex corrections, the Onsager coefficients are given by (denoting for simplicity $L_{11} \equiv L_{0}, L_{12} \equiv L_{1}, L_{22} \equiv L_{2}$ )

$$
L_{n}=\frac{1}{T^{n}} \int d \omega\left(-\frac{\partial f}{\partial \omega}\right) \omega^{n} \mathcal{T}(\omega),
$$

in which $f(\omega)=1 /\left[1+e^{\omega / T}\right]$ is the Fermi function and

$$
\mathcal{T}(\omega)=2 \pi \int \frac{d^{d} k}{(2 \pi)^{d}} v_{\mathbf{k}}^{2} A(\mathbf{k}, \omega)^{2} .
$$

In this expression, $v_{\mathbf{k}}=\left(\nabla_{\mathbf{k}} \varepsilon_{\mathbf{k}}\right)_{\alpha} / \hbar$ denotes the band velocity in the direction $\alpha=x, y, z$ being considered, and $A(\mathbf{k}, \omega)$ is the electronic spectral function, related to the self-energy $\Sigma=$ $\Sigma\left(\omega+i 0^{+}, \mathbf{k}\right)$ by

$$
A(\mathbf{k}, \omega)=\frac{1}{\pi} \frac{\Gamma / 2}{\left(\omega+\mu-\varepsilon_{\mathbf{k}}-\operatorname{Re} \Sigma\right)^{2}+(\Gamma / 2)^{2}} .
$$

In this expression, $\Sigma=\Sigma\left(\omega+i 0^{+}, \mathbf{k}\right)$ is the self-energy due to inelastic interactions between electrons, and $\Gamma$ is the full scattering rate including both elastic and inelastic terms:

$$
\Gamma(\mathbf{k}, \omega)=\gamma_{\mathbf{k}}-2 \operatorname{Im} \Sigma\left(\mathbf{k}, \omega+i 0^{+}\right) .
$$

These expressions can be further simplified when the elastic scattering rate $\gamma_{\mathbf{k}}$ and electron-electron self-energy $\Sigma$ do not depend on momentum and when the vertex corrections can be neglected [23].

Using the band transport function $\Phi$, one can express

$$
\mathcal{T}(\omega)=\pi \int d \varepsilon \Phi(\varepsilon) A(\varepsilon, \omega)^{2} .
$$

We change the integration variable by setting $\varepsilon=\omega+\mu-$ $\operatorname{Re} \Sigma+\frac{\Gamma}{2} y$, so that

$$
\mathcal{T}(\omega)=\pi \frac{2}{\Gamma} \int_{-\infty}^{+\infty} d y \Phi\left(\omega+\mu-\operatorname{Re} \Sigma+\frac{\Gamma}{2} y\right)\left(\frac{1 / \pi}{y^{2}+1}\right)^{2} .
$$

We now perform an expansion of this expression for small $\Gamma$ and retain only the most singular term, yielding

$$
\mathcal{T}(\omega)=\frac{1}{\Gamma} \Phi(\omega+\mu-\operatorname{Re} \Sigma)+\cdots,
$$

where we have used $\int_{-\infty}^{+\infty} d y 1 / \pi^{2}\left(y^{2}+1\right)^{2}=1 /(2 \pi)$. It is convenient to introduce the notations

$$
\begin{aligned}
\bar{\varepsilon}_{F}(T) & \equiv \mu-\operatorname{Re} \Sigma(\omega=0, T), \\
1-\frac{1}{Z(T, \omega)} & \equiv \frac{1}{\omega}[\operatorname{Re} \Sigma(\omega, T)-\operatorname{Re} \Sigma(0, T)],
\end{aligned}
$$


so that, to dominant order in $\Gamma$,

$$
\mathcal{T}(\omega)=\frac{1}{\Gamma(T, \omega)} \Phi\left[\bar{\varepsilon}_{F}(T)+\frac{\omega}{Z(T, \omega)}\right]+\cdots .
$$

Inserting this expression in the Onsager coefficients $L_{n}$ above, and changing the variable to $x=\omega / T$ in the integral over frequency, we obtain, with $\tau=1 / \Gamma$,

$$
L_{n}=\int d x \frac{x^{n}}{4 \cosh ^{2} \frac{x}{2}} \Phi\left[\bar{\varepsilon}_{F}(T)+x \frac{T}{Z(T, x T)}\right] \tau(T, x T) .
$$

We now perform a low- $T$ expansion of this expression. We note that for both the Fermi-liquid and non-Fermi-liquid cases considered in this paper, $T / Z(T, x T)$ vanishes at low $T$ (as $\sim T$ and $\sim T^{v}$, respectively). We assume furthermore that the $T$ dependence of $\bar{\varepsilon}_{F}(T)$ is subdominant and can be neglected. We thus obtain

$$
L_{n}=\Phi_{0}\left\langle x^{n} \tau(T, x T)\right\rangle+T \Phi_{0}^{\prime}\left\langle x^{n+1} \frac{\tau(T, x T)}{Z(T, x T)}\right\rangle+\cdots,
$$

with the notation

$$
\langle F(x)\rangle=\int_{-\infty}^{+\infty} d x \frac{F(x)}{4 \cosh ^{2} \frac{x}{2}} .
$$

Retaining just the lowest two terms in the Taylor expansion of $\phi(\varepsilon)$ is sufficient at low temperatures as the transport function usually varies only on the bare electronic scales, e.g., the bandwidth. We have checked furthermore that neglecting the frequency $(x)$ dependence of $Z$ in these equations is a good approximation, so that we finally obtain the expressions (4)-(6) of Sec. II.

We define $I_{n}=L_{n} / \Phi_{0}$ and note the expression of the conductivity:

$$
\sigma=e^{2} \Phi_{0} I_{0}(T) \simeq e^{2} \Phi_{0}\left\langle\tau_{+}\right\rangle .
$$

Although the non-Fermi-liquid case does not have conventional quasiparticles, the final expressions for $S, \sigma$, and the Onsager coefficients $L_{n}$ at low $T$ are formally identical to the ones obtained by applying a Boltzmann equation formalism to excitations with a lifetime $\tau^{\mathrm{ex}}=\tau / Z$ and dispersing as $\varepsilon_{\mathbf{k}}^{\mathrm{ex}}=$ $Z \varepsilon_{\mathbf{k}}$, corresponding to a transport function of these excitations $\Phi_{e x}(\varepsilon)=Z \Phi(\varepsilon / Z)$.

In Fig. 4, we compare the Seebeck coefficient evaluated with the full Kubo formula and with the simplified Boltzmann-like expressions. The data are for the subPlanckian $v=1 / 2$ case. The results of the full Kubo evaluation (dotted curves) agree well with the results of a simplified Boltzmann calculation that approximates $Z(\omega, T)$ by its zero-frequency value $Z(\omega, T) \rightarrow Z(0, T)$ (solid curves). The result of such Boltzmann calculations are the data given in the main text. The remaining small discrepancy between the Kubo and the Boltzmann results can be remedied if one retains the frequency dependence of $Z(\omega, T)$ (dashed curves that overlap with the dotted ones).

For application of our theory to cuprates it is essential to retain momentum dependence. However, as recently reported in an angular-dependent magnetoresistance (ADMR) study of cuprates [20], the inelastic scattering is found to be local, and it is sufficient to retain the momentum dependence in the elastic scattering rate. In such a case, the theory can be

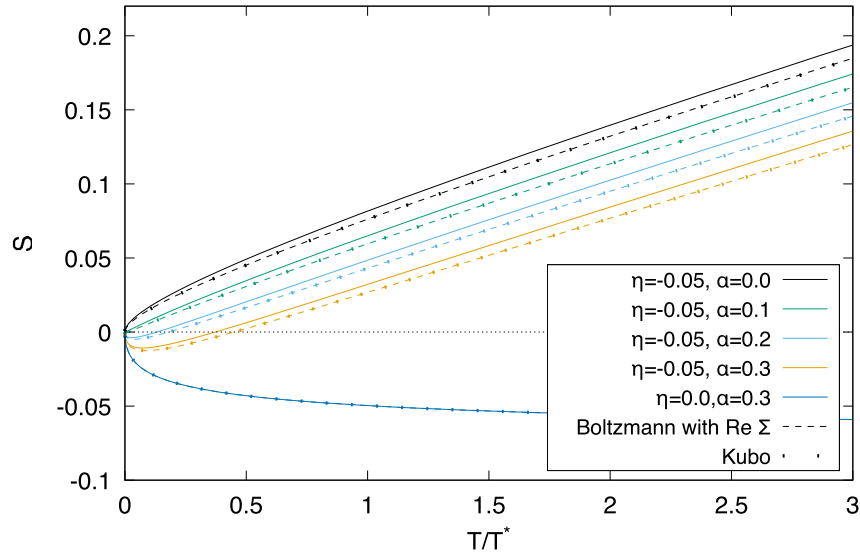

FIG. 4. Seebeck coefficient in units of $k_{B} / e$ for the $v=1 / 2$ case. $\pi T^{*} / \gamma=1$. The results of the Boltzmann calculation with $Z=Z(T)$ (solid curves) are compared with the Kubo calculation (dotted curves) and a Boltzmann calculation that retains the frequency dependence of $Z=Z(\omega, T)$ (dashed curves). The results of the latter two cannot be distinguished on the scale of this plot. Similar agreement between the Boltzmann and Kubo results is found for all the data in the main text.

minimally extended, with only quantitative but no qualitative changes, by allowing for the elastic rate to have momentum dependence. Such an extension is presented in Ref. [59].

\section{APPENDIX B: SKEWED NON-FERMI LIQUIDS AND $\omega / T$ SCALING}

\section{Scaling function and conformally invariant case}

The (local) non-Fermi liquids considered in this paper have a scattering rate that obeys $\omega / T$ scaling $(\nu \leqslant 1)$ :

$$
\Gamma_{\text {in }}(T, \omega)=\lambda(\pi T)^{v} g\left(\frac{\omega}{T}\right) .
$$

The scaling function $g(x)$ has a regular expansion at small $x$ : $g(x)=g(0)+x g^{\prime}(0)+\cdots$, while at large $x$ it obeys $g(x \rightarrow$ $+\infty) \sim c_{+}^{\infty} x^{v}$ and $g(x \rightarrow-\infty) \sim c_{-}^{\infty}|x|^{\nu}$. The "skewed" case with a particle-hole asymmetry will in general have $g^{\prime}(0) \neq 0$ and $c_{+}^{\infty} \neq c_{-}^{\infty}$. Hence

$$
\begin{aligned}
& \Gamma_{\text {in }}(T \gg|\omega|) \sim \lambda g(0)(\pi T)^{v}+\lambda g^{\prime}(0) \frac{\pi \omega}{(\pi T)^{1-v}}+\cdots, \\
& \Gamma_{\text {in }}(T \ll|\omega|) \sim c_{ \pm}^{\infty} \lambda|\pi \omega|^{v}+\cdots .
\end{aligned}
$$

Our qualitative results do not depend on the specific form of the scaling function $g(x)$ provided it obeys these general properties.

In Sec. III we considered $g(x)$ given by Eq. (11). It has been shown in Ref. [36], in the context of overscreened Kondo impurity models, that this specific form of the scaling function holds in models which have conformal invariance at low energy or temperature. It is also relevant in the context of SYK models $[39-42,46]$. The parameter $\alpha$ controls the particle-hole asymmetry (see Fig. 5), with $\alpha=0$ corresponding to particlehole symmetry $g(x)=g(-x)$ and $g_{-\alpha, v}(x)=g_{\alpha, v}(-x)$. The normalization $g_{\alpha=0, v}(0)=1$ was chosen in the above expression. In the Planckian case $v=1$, Eq. (11) can be cast in the 


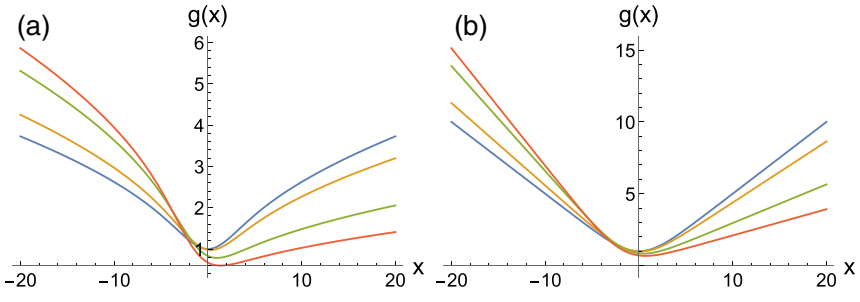

FIG. 5. Scaling function $g_{\alpha, v}(x)$ for $v=1 / 2$ (a) and $v=1$ (b), for different values of the asymmetry parameter $\alpha=0,0.3,1,1.5$ (blue, yellow, green, and red).

explicit form

$$
\begin{aligned}
g_{\alpha, \nu=1}(x) & =\frac{(x+\alpha) / 2}{\sinh [(x+\alpha) / 2]} \frac{\cosh (x / 2)}{\cosh (\alpha / 2)}, \\
\Gamma_{\text {in }}(T, \omega) & =\lambda \pi T \frac{(\omega / T+\alpha) / 2}{\sinh [(\omega / T+\alpha) / 2]} \frac{\cosh (\omega / 2 T)}{\cosh (\alpha / 2)} .
\end{aligned}
$$

The connection between this scaling form and conformal invariance in local models is the following. This invariance implies that any imaginary time $(\tau)$ fermionic correlation function (for example, the self-energy) takes the following form in the limit where $\tau$ and inverse temperature $\beta=1 / T$ are both large compared with microscopic scales, but with arbitrary $\tau / \beta[36]$ :

$$
\Sigma(\tau) \propto e^{\alpha(\tau / \beta-1 / 2)}\left(\frac{\pi / \beta}{\sin \pi \tau / \beta}\right)^{1+v} .
$$

This function has the following spectral representation $(\bar{\tau}=\tau / \beta)$ :

$$
e^{\alpha(\bar{\tau}-1 / 2)}\left(\frac{\pi}{\sin \pi \bar{\tau}}\right)^{1+v}=C \int_{-\infty}^{+\infty} d x \frac{e^{-x \bar{\tau}}}{1+e^{-x}} g_{\alpha, v}(x),
$$

with $C=\cosh (\alpha / 2)(2 \pi)^{\nu} \Gamma[(1+v) / 2]^{2} /(\pi \Gamma[1+\nu])$.

Other forms of the scaling functions $g$ can also be considered. In the absence of a particle-hole asymmetry, the following phenomenological scaling function has sometimes been considered in the literature (see, e.g., Ref. [54]):

$$
g_{\alpha=0}(x)=\left[1+x^{2} / \pi^{2}\right]^{\nu / 2} .
$$

Note that $v=2$ reduces to the Fermi-liquid scaling function $1+x^{2} / \pi^{2}$ : This is also the case of Eq. (11) for $\nu=2, \alpha=0$. Starting with this form, one can introduce an asymmetry, for example, by deforming the scaling function in an analogous way to Eq. (11) [36]:

$$
g_{\alpha}(x)=g_{0}(x+\alpha) \frac{\cosh (x / 2)}{\cosh [(x+\alpha) / 2] \cosh (\alpha / 2)} .
$$

A simple calculation using the spectral representation of $\Sigma$ then shows that

$$
\Sigma_{\alpha}(\tau)=\frac{e^{\alpha(\tau / \beta-1 / 2)}}{\cosh \alpha / 2} \Sigma_{\alpha=0}(\tau) .
$$

\section{Scaling form of $Z(T, \omega)$}

Here, we discuss the scaling form of the real part of the self-energy. We start from the spectral representation

$$
\begin{aligned}
\operatorname{Re} \Sigma(\omega) & =P \int d \epsilon \frac{\sigma(\epsilon)}{\omega-\epsilon}, \\
\sigma(\omega) & =-\frac{1}{\pi} \operatorname{Im} \Sigma\left(\omega+i 0^{+}\right)=\frac{1}{2 \pi} \Gamma_{\mathrm{in}}(T, \omega),
\end{aligned}
$$

in which $P$ denotes the principal part of the integral and we have recalled the relation (factor of 2 ) between the imaginary part of the self-energy and the inelastic scattering rate for a local theory. The frequency- and temperature-dependent effective mass enhancement $1 / Z(T, \omega)$ is given by

$$
1-\frac{1}{Z(T, \omega)} \equiv \frac{1}{\omega}[\operatorname{Re} \Sigma(\omega)-\operatorname{Re} \Sigma(0)]=P \int d \epsilon \frac{\sigma(\epsilon)}{\epsilon(\omega-\epsilon)} .
$$

Because we have subtracted $\operatorname{Re} \Sigma(0)$, we can substitute the scaling form of $\sigma=\Gamma_{\text {in }} / 2$ in the integral without encountering divergencies for a non-Fermi liquid with $v<1$, and thus we obtain

$$
\frac{T}{Z(T, \omega=x T)}=T-\frac{\lambda}{2 \pi}(\pi T)^{v} P \int d y \frac{g(y)}{y(x-y)} .
$$

Hence, remarkably, $T$ / $Z$ obeys a universal scaling form which depends only on the scaling function $g$. This is in contrast to a Fermi liquid in which $Z$ depends on all energy scales [as signaled by the fact that inserting the low-energy expression $\sigma(\omega) \propto \omega^{2}+(\pi T)^{2}$ in Eq. (B9) would lead to a divergent integral].

For results given in Sec. III, we ignore the $\omega$ (i.e., $x$ ) dependence of $Z$ and replace $Z(T, \omega)$ by $Z(T, 0)=Z(T)$. Comparison with the full Kubo formula calculations given in Fig. 4 justifies this approximation for transport calculations. The $x \rightarrow 0$ limit of the above expression requires some care because of the appearance of a double pole. This is resolved by integrating by parts, and one obtains

$$
\frac{T}{Z(T, \omega=0)}=T+\frac{\lambda}{2 \pi}(\pi T)^{\nu} \int d y \frac{g^{\prime}(y)}{y},
$$

so that, with the notation introduced in the text,

$$
\frac{T}{Z(T)}=T+\frac{\lambda(\pi T)^{v}}{c_{Z}}, \quad \frac{1}{c_{Z}}=\frac{1}{2 \pi} \int d y \frac{g^{\prime}(y)}{y} .
$$

These expressions are valid for $v<1$. The Planckian case $v=1$ requires a slightly different analysis because in Eq. (B9) the integral would diverge logarithmically at large frequency. Hence a cutoff $\Lambda$ must be kept. The low-temperature behavior of the zero-frequency limit of $Z(T)$ can be again obtained from an integration by part (up to the cutoff). We obtain

$$
1-1 / Z(T)=-\frac{\lambda}{2}\left[P \int_{-\Lambda / T}^{+\Lambda / T} d y \frac{g^{\prime}(y)}{y}-\left.\frac{g(y)}{y}\right|_{-\Lambda / T} ^{\Lambda / T}\right] .
$$

At large $y, g^{\prime}$ tends to a constant in the Planckian case $g^{\prime}(y \sim$ $\pm \infty) \rightarrow g^{\prime}( \pm \infty)$, so that the low-temperature behavior is

$$
\frac{1}{Z(T)}=1+\frac{\lambda}{2}\left[g^{\prime}(+\infty)-g^{\prime}(-\infty)\right] \ln \left(\zeta \frac{\Lambda}{T}\right),
$$




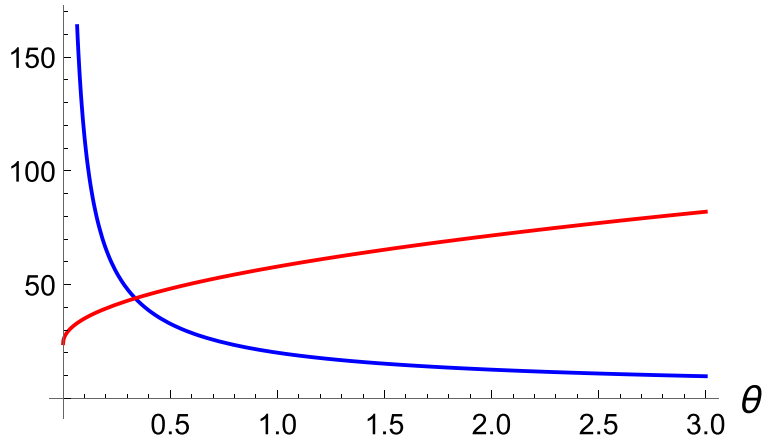

FIG. 6. $\theta^{v} F_{2}(\theta) / F_{1}(\theta)$ vs $\theta$ (red) and $F_{0}(\theta) / \theta^{v} F_{1}(\theta)$ vs $\theta$ (blue) for $v=0.5$ and $\alpha=0.3$.

with $\zeta \simeq 0.28$ being a numerically determined constant. Hence, using the notation in the main text (and absorbing $\zeta$ in the definition of the cutoff $\Lambda$ ), we obtain $2 \pi / c_{Z}=$ $\left[g^{\prime}(+\infty)-g^{\prime}(-\infty)\right](=1$ for any $\alpha)$. In the Planckian case, the high-energy cutoff does not entirely disappear from the expression of $Z$, but we note that the prefactor of the $\ln T$ term depends only on the inelastic coupling constant and not on the cutoff.

\section{APPENDIX C: TEMPERATURE DEPENDENCE OF THE SEEBECK COEFFICIENT}

Setting $\theta=T / T^{*}$ and $\eta=\gamma \Phi_{0}^{\prime} / \Phi_{0}$, expressions (5) and (6) can be written as

$$
\begin{aligned}
& I_{1}(\theta)=F_{1}(\theta)+\eta\left[\frac{T^{*}}{\gamma} \theta+\frac{1}{c_{Z}} \theta^{\nu}\right] F_{2}(\theta), \\
& I_{0}(\theta)=F_{0}(\theta)+\eta\left[\frac{T^{*}}{\gamma} \theta+\frac{1}{c_{Z}} \theta^{\nu}\right] F_{1}(\theta),
\end{aligned}
$$

with

$$
F_{n}(\theta)=\left\langle\frac{x^{n}}{1+\theta^{v} g(x)}\right\rangle .
$$

The low-temperature expansion $(\theta \rightarrow 0)$ of the functions $F_{n}$ reads

$$
\begin{aligned}
& F_{0}(\theta)=1-\langle g\rangle \theta^{v}+\left\langle g^{2}\right\rangle \theta^{2 v}+\cdots, \\
& F_{1}(\theta)=-\langle x g\rangle \theta^{v}+\left\langle x g^{2}\right\rangle \theta^{2 v}+\cdots, \\
& F_{2}(\theta)=\frac{\pi^{2}}{3}-\left\langle x^{2} g\right\rangle \theta^{v}+\left\langle x^{2} g^{2}\right\rangle \theta^{2 v}+\cdots .
\end{aligned}
$$

The key difference in the behavior of $F_{n}$ is that $F_{1} \sim c_{-} \theta^{v}$ $\left(c_{-}=\langle x g\rangle\right)$ while $F_{0,2} \sim$ const. As a result, $\theta^{v} F_{2}$ and $F_{1}$ have

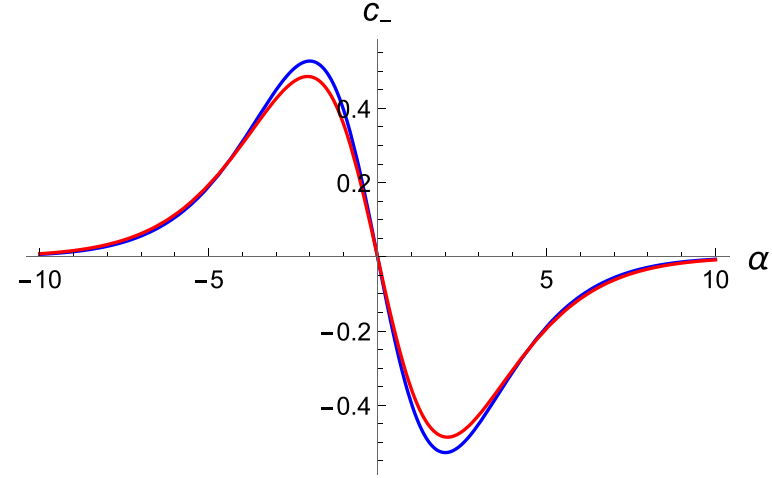

FIG. 7. Coefficient $c_{-}$vs $\alpha$ for $v=0.5$ (blue) and $v=1$ (red).

the same temperature dependence in this regime, and hence both contribute to $I_{1}$ and to the thermopower. In contrast, in $I_{0}$, the term $\theta^{v} F_{1} \sim \theta^{2 v}$ can be neglected in comparison to $F_{0}$ in both the conductivity and the Seebeck coefficient, which can be hence written as

$$
S(T)=-\frac{k_{B}}{e}\left[\eta\left(\frac{1}{c_{Z}} \theta^{v}+\frac{T^{*}}{\gamma} \theta\right) \frac{F_{2}(\theta)}{F_{0}(\theta)}+\frac{F_{1}(\theta)}{F_{0}(\theta)}\right] .
$$

$F_{1}$ is much smaller in magnitude than $\theta^{v} F_{2}$, as shown in Fig. 6. Hence the odd-frequency contribution $F_{1}$ in $I_{1}$ is comparable to the even one $\eta \theta^{v} F_{2}$ only when the parameter $\eta=\gamma \Phi_{0}^{\prime} / \Phi_{0}$ is small (of order $10^{-2}$ ). This is, however, typically the case since the elastic scattering rate is usually much smaller than electronic energy scales (the elastic scattering rate is usually much smaller than the bandwidth). Hence, at low $T$, the Seebeck coefficient reads, for $v<1$,

$$
S \sim-\frac{k_{B}}{e}\left(\frac{T}{T^{*}}\right)^{v}\left[\frac{\pi^{2}}{3 c_{Z}} \eta-c_{-}\right] .
$$

The dependence of the coefficient $c_{-}$on the asymmetry parameter $\alpha$ for the choice [Eq. (11)] of scaling functions is displayed in Fig. 7.

In the high-temperature limit $(\theta \rightarrow \infty)$, all the functions $F_{n}$ have the same temperature dependence $\sim c_{n} / \theta^{\nu}$ :

$$
F_{n}(\theta)=\frac{1}{\theta^{v}}\left\langle\frac{x^{n}}{g}\right\rangle-\frac{1}{\theta^{2 v}}\left\langle\frac{x^{n}}{g^{2}}\right\rangle+\cdots .
$$

Hence, formally, at very high $T, S$ tends to a constant $=-c_{2} / c_{1} k_{B} / e$ in the presence of odd-frequency scattering. However, the term involving $F_{1}$ in $I_{0}$ remains small for most temperatures of interest as shown in Fig. 6. Hence, in practice, the relevant high- $T$ behavior of $S$ is

$$
S \sim-\frac{k_{B}}{e}\left[\frac{c_{2}}{c_{0}} \frac{\Phi_{0}^{\prime}}{\Phi_{0}} T+\eta \frac{c_{2}}{c_{0} c_{Z}}\left(\frac{T}{T^{*}}\right)^{v}+\frac{c_{1}}{c_{0}}\right] .
$$

[1] H. J. Goldsmid, Introduction to Thermoelectricity (Springer, New York, 2009).

[2] K. Behnia, Fundamentals of Thermoelectricity (Oxford University Press, Oxford, 2015).

[3] A. Georges, https://www.college-de-france.fr/site/en-antoinegeorges/index.htm, Lectures at the Collège de France 20122013 and 2013-2014.
[4] J. E. Robinson, Thermoelectric power in the nearly-free-electron model, Phys. Rev. 161, 533 (1967).

[5] K. Haule and G. Kotliar, Thermoelectrics near the Mott localization-delocalization transition, in Properties and Applications of Thermoelectric Materials, edited by V. Zlatic and A. C. Hewson (Springer, Netherlands, 2009), pp. 119-131. 
[6] G.-H. Gweon, B. S. Shastry, and G. D. Gu, Extremely Correlated Fermi-Liquid Description of Normal-State ARPES in Cuprates, Phys. Rev. Lett. 107, 056404 (2011).

[7] B. S. Shastry, Dynamical Particle-Hole Asymmetry in HighTemperature Cuprate Superconductors, Phys. Rev. Lett. 109, 067004 (2012).

[8] X. Deng, J. Mravlje, R. Žitko, M. Ferrero, G. Kotliar, and A. Georges, How Bad Metals Turn Good: Spectroscopic Signatures of Resilient Quasiparticles, Phys. Rev. Lett. 110, 086401 (2013).

[9] W. Xu, K. Haule, and G. Kotliar, Hidden Fermi Liquid, Scattering Rate Saturation, and Nernst Effect: A Dynamical Mean-Field Theory Perspective, Phys. Rev. Lett. 111, 036401 (2013).

[10] R. Žitko, D. Hansen, E. Perepelitsky, J. Mravlje, A. Georges, and B. S. Shastry, Extremely correlated Fermi liquid theory meets dynamical mean-field theory: Analytical insights into the doping-driven Mott transition, Phys. Rev. B 88, 235132 (2013).

[11] M. Shahbazi and C. Bourbonnais, Seebeck coefficient in correlated low-dimensional organic metals, Phys. Rev. B 94, 195153 (2016).

[12] R. Žitko, H. R. Krishnamurthy, and B. S. Shastry, Reversal of particle-hole scattering-rate asymmetry in the Anderson impurity model, Phys. Rev. B 98, 161121(R) (2018).

[13] B. Xu and M. J. Verstraete, First Principles Explanation of the Positive Seebeck Coefficient of Lithium, Phys. Rev. Lett. 112, 196603 (2014).

[14] B. Xu, M. Di Gennaro, and M. J. Verstraete, Thermoelectric properties of elemental metals from first-principles electron-phonon coupling, Phys. Rev. B 102, 155128 (2020).

[15] J. Zaanen, Superconductivity: Why the temperature is high, Nature (London) 430, 512 (2004).

[16] D. van der Marel, H. J. A. Molegraaf, J. Zaanen, Z. Nussinov, F. Carbone, A. Damascelli, H. Eisaki, M. Greven, P. H. Kes, and M. Li, Quantum critical behaviour in a high- $T_{\mathrm{c}}$ superconductor, Nature (London) 425, 271 (2003).

[17] J. A. N. Bruin, H. Sakai, R. S. Perry, and A. P. Mackenzie, Similarity of scattering rates in metals showing T-linear resistivity, Science 339, 804 (2013).

[18] S. A. Hartnoll, Theory of universal incoherent metallic transport, Nat. Phys. 11, 54 (2014).

[19] A. Legros, S. Benhabib, W. Tabis, F. Laliberte, M. Dion, M. Lizaire, B. Vignolle, D. Vignolles, H. Raffy, Z. Z. Li, P. Auban-Senzier, N. Doiron-Leyraud, P. Fournier, D. Colson, L. Taillefer, and C. Proust, Universal T-linear resistivity and Planckian dissipation in overdoped cuprates, Nat. Phys. 15, 142 (2019).

[20] G. Grissonnanche, Y. Fang, A. Legros, S. Verret, F. Laliberté, C. Collignon, J. Zhou, D. Graf, P. Goddard, L. Taillefer, and B. J. Ramshaw, Linear-in temperature resistivity from an isotropic Planckian scattering rate, Nature (London) 595, 667 (2021).

[21] C. M. Varma, Colloquium: Linear in temperature resistivity and associated mysteries including high temperature superconductivity, Rev. Mod. Phys. 92, 031001 (2020).

[22] B. Michon, C. Girod, S. Badoux, J. Kačmarčík, Q. Ma, M. Dragomir, H. A. Dabkowska, B. D. Gaulin, J.-S. Zhou, S. Pyon, T. Takayama, H. Takagi, S. Verret, N. Doiron-Leyraud,
C. Marcenat, L. Taillefer, and T. Klein, Thermodynamic signatures of quantum criticality in cuprate superconductors, Nature (London) 567, 218 (2019).

[23] A. Khurana, Electrical Conductivity in the Infinite-Dimensional Hubbard Model, Phys. Rev. Lett. 64, 1990 (1990).

[24] G. Uhrig and D. Vollhardt, Drude weight and dc conductivity of correlated electrons, Phys. Rev. B 52, 5617 (1995).

[25] J. Vučičević, J. Kokalj, R. Žitko, N. Wentzell, D. Tanasković, and J. Mravlje, Conductivity in the Square Lattice Hubbard Model at High Temperatures: Importance of Vertex Corrections, Phys. Rev. Lett. 123, 036601 (2019).

[26] A. Kauch, P. Pudleiner, K. Astleithner, P. Thunström, T. Ribic, and K. Held, Generic Optical Excitations of Correlated Systems: $\pi$-tons, Phys. Rev. Lett. 124, 047401 (2020).

[27] A. Vranić, J. Vučičević, J. Kokalj, J. Skolimowski, R. Žitko, J. Mravlje, and D. Tanasković, Charge transport in the Hubbard model at high temperatures: Triangular versus square lattice, Phys. Rev. B 102, 115142 (2020).

[28] O. Simard, S. Takayoshi, and P. Werner, Diagrammatic study of optical excitations in correlated systems, Phys. Rev. B 103, 104415 (2021).

[29] P. Worm, C. Watzenböck, M. Pickem, A. Kauch, and K. Held, Broadening and sharpening of the Drude peak through antiferromagnetic fluctuations, Phys. Rev. B 104, 115153 (2021).

[30] A. Lavasani, D. Bulmash, and S. Das Sarma, Wiedemann-Franz law and Fermi liquids, Phys. Rev. B 99, 085104 (2019).

[31] S. Li, Z. Tong, X. Zhang, and H. Bao, Thermal conductivity and Lorenz ratio of metals at intermediate temperatures with mode-level first-principles analysis, Phys. Rev. B 102, 174306 (2020).

[32] Everywhere in the main text it is understood that the temperature is low compared with the energy over which $\Phi$ varies, e.g., compared with the energy scale $\Phi_{0} / \Phi_{0}^{\prime}$. Otherwise the low- $T$ expansion leading to (5) and (6) is no longer valid, and the full Kubo formula detailed in Appendix A has to be used.

[33] $Z(T)$ is finite at $T \neq 0$ even when the low-frequency behavior of the self-energy is singular at $T=0$.

[34] I. Paul and G. Kotliar, Thermoelectric behavior near the magnetic quantum critical point, Phys. Rev. B 64, 184414 (2001).

[35] K. Behnia, D. Jaccard, and J. Flouquet, On the thermoelectricity of correlated electrons in the zero-temperature limit, J. Phys.: Condens. Matter 16, 5187 (2004).

[36] O. Parcollet, A. Georges, G. Kotliar, and A. Sengupta, Overscreened multichannel SU( $n)$ Kondo model: Large- $n$ solution and conformal field theory, Phys. Rev. B 58, 3794 (1998).

[37] S. Sachdev and J. Ye, Gapless Spin-Fluid Ground State in a Random Quantum Heisenberg Magnet, Phys. Rev. Lett. 70, 3339 (1993).

[38] A. Kitaev, A simple model of quantum holography, talk given at the KITP program "Entanglement in Strongly-Correlated Quantum Matter," 2015.

[39] A. Georges, O. Parcollet, and S. Sachdev, Quantum fluctuations of a nearly critical Heisenberg spin glass, Phys. Rev. B 63, 134406 (2001).

[40] O. Parcollet and A. Georges, Non-Fermi-liquid regime of a doped Mott insulator, Phys. Rev. B 59, 5341 (1999).

[41] S. Sachdev, Bekenstein-Hawking Entropy and Strange Metals, Phys. Rev. X 5, 041025 (2015).

[42] R. A. Davison, W. Fu, A. Georges, Y. Gu, K. Jensen, and S. Sachdev, Thermoelectric transport in disordered metals without 
quasiparticles: The Sachdev-Ye-Kitaev models and holography, Phys. Rev. B 95, 155131 (2017).

[43] M. Tikhanovskaya, H. Guo, S. Sachdev, and G. Tarnopolsky, Excitation spectra of quantum matter without quasiparticles. I. Sachdev-Ye-Kitaev models, Phys. Rev. B 103, 075141 (2021).

[44] M. Tikhanovskaya, H. Guo, S. Sachdev, and G. Tarnopolsky, Excitation spectra of quantum matter without quasiparticles. II. Random $t$ - $J$ models, Phys. Rev. B 103, 075142 (2021).

[45] A. Kruchkov, A. A. Patel, P. Kim, and S. Sachdev, Thermoelectric power of Sachdev-Ye-Kitaev islands: Probing BekensteinHawking entropy in quantum matter experiments, Phys. Rev. B 101, 205148 (2020).

[46] P. Dumitrescu, N. Wentzell, A. Georges, and O. Parcollet, Planckian metal at a doping-induced quantum critical point, arXiv:2103.08607.

[47] S. Sachdev, Quantum Phase Transitions, 2nd ed. (Cambridge University Press, Cambridge, 2011).

[48] J. M. Buhmann, M. Ossadnik, T. M. Rice, and M. Sigrist, Numerical study of charge transport of overdoped $\mathrm{La}_{2-x} \mathrm{Sr}_{x} \mathrm{CuO}_{4}$ within semiclassical Boltzmann transport theory, Phys. Rev. B 87, 035129 (2013).

[49] A. Varlamov, V. Egorov, and A. Pantsulaya, Kinetic properties of metals near electronic topological transitions (2 1/2-order transitions), Adv. Phys. 38, 469 (1989).

[50] F. Herman, J. Buhmann, M. H. Fischer, and M. Sigrist, Deviation from Fermi-liquid transport behavior in the vicinity of a Van Hove singularity, Phys. Rev. B 99, 184107 (2019).

[51] N. E. Hussey, Phenomenology of the normal state in-plane transport properties of high- $T_{\mathrm{c}}$ cuprates, J. Phys.: Condens. Matter 20, 123201 (2008).

[52] L. Taillefer, Scattering and pairing in cuprate superconductors, Annu. Rev. Condens. Matter Phys. 1, 51 (2010).

[53] C. C. Homes, S. V. Dordevic, M. Strongin, D. A. Bonn, R. Liang, W. N. Hardy, S. Komiya, Y. Ando, G. Yu, N. Kaneko, X. Zhao, M. Greven, D. N. Basov, and T. Timusk, A universal scaling relation in high-temperature superconductors, Nature (London) 430, 539 (2004).

[54] T. J. Reber, X. Zhou, N. C. Plumb, S. Parham, J. A. Waugh, Y. Cao, Z. Sun, H. Li, Q. Wang, J. S. Wen, Z. J. Xu, G. Gu, Y. Yoshida, H. Eisaki, G. B. Arnold, and D. S. Dessau, A unified form of low-energy nodal electronic interactions in hole-doped cuprate superconductors, Nat. Commun. 10, 5737 (2019).

[55] F. Laliberté, J. Chang, N. Doiron-Leyraud, E. Hassinger, R. Daou, M. Rondeau, B. J. Ramshaw, R. Liang, D. A. Bonn, W. N. Hardy, S. Pyon, T. Takayama, H. Takagi, I. Sheikin, L. Malone, C. Proust, K. Behnia, and L. Taillefer, Fermi-surface reconstruction by stripe order in cuprate superconductors, Nat. Commun. 2, 432 (2011).

[56] R. Daou, O. Cyr-Choinière, F. Laliberté, D. LeBoeuf, N. Doiron-Leyraud, J.-Q. Yan, J.-S. Zhou, J. B. Goodenough, and L. Taillefer, Thermopower across the stripe critical point of $\mathrm{La}_{1.6-x} \mathrm{Nd}_{0.4} \mathrm{Sr}_{x} \mathrm{CuO}_{4}$ : Evidence for a quantum critical point in a hole-doped high- $T_{c}$ superconductor, Phys. Rev. B 79, 180505(R) (2009).

[57] C. Collignon, A. Ataei, A. Gourgout, S. Badoux, M. Lizaire, A. Legros, S. Licciardello, S. Wiedmann, J. Q. Yan, J. S. Zhou, Q. Ma, B. D. Gaulin, N. Doiron-Leyraud, and L. Taillefer, Thermopower across the phase diagram of the cuprate $\mathrm{La}_{1.6-x} \mathrm{Nd}_{0.4} \mathrm{Sr}_{x} \mathrm{CuO}_{4}$ : Signatures of the pseudogap and charge density wave phases, Phys. Rev. B 103, 155102 (2021).
[58] S. Verret, O. Simard, M. Charlebois, D. Sénéchal, and A.-M. S. Tremblay, Phenomenological theories of the low-temperature pseudogap: Hall number, specific heat, and Seebeck coefficient, Phys. Rev. B 96, 125139 (2017).

[59] A. Gourgout, G. Grissonnanche, F. Laliberté, A. Ataei, L. Chen, S. Verret, J.-S. Zhou, J. Mravlje, A. Georges, N. DoironLeyraud, and L. Taillefer, Seebeck coefficient in a cuprate superconductor: particle-hole asymmetry in the strange metal phase and Fermi surface transformation in the pseudogap phase, arXiv:2106.05959.

[60] M. Lizaire, A. Legros, A. Gourgout, S. Benhabib, S. Badoux, F. Laliberté, M. E. Boulanger, A. Ataei, G. Grissonnanche, D. LeBoeuf, S. Licciardello, S. Wiedmann, S. Ono, H. Raffy, S. Kawasaki, G. Q. Zheng, N. Doiron-Leyraud, C. Proust, and L. Taillefer, Transport signatures of the pseudogap critical point in the cuprate superconductor $\mathrm{Bi}_{2} \mathrm{Sr}_{2-x} \mathrm{La}_{x} \mathrm{CuO}_{6+\delta}$ Phys. Rev. B 104, 014515 (2021)

[61] H. Jin, A. Narduzzo, M. Nohara, H. Takagi, N. E. Hussey, and K. Behnia, Positive Seebeck coefficient in highly doped $\mathrm{La}_{2-x} \mathrm{Sr}_{x} \mathrm{CuO}_{4}$ at $x=0.33$; its origin and implication, J. Phys. Soc. Jpn. 90, 053702 (2021).

[62] Y. Nakamura and S. Uchida, Anisotropic transport properties of single-crystal $\mathrm{La}_{2-x} \mathrm{Sr}_{x} \mathrm{CuO}_{4}$ : Evidence for the dimensional crossover, Phys. Rev. B 47, 8369 (1993).

[63] J.-S. Zhou and J. B. Goodenough, Thermoelectric power in single-layer copper oxides, Phys. Rev. B 51, 3104 (1995).

[64] S. Nakamae, K. Behnia, N. Mangkorntong, M. Nohara, H. Takagi, J. C. Yates, and E. Hussey, Electronic ground state of heavily overdoped nonsuperconducting $\mathrm{La}_{2-x} \mathrm{Sr}_{x} \mathrm{CuO}_{4}$, Phys. Rev. B 68, 100502(R) (2003).

[65] C. G. Fatuzzo, Y. Sassa, M. Månsson, S. Pailhès, O. J. Lipscombe, S. M. Hayden, L. Patthey, M. Shi, M. Grioni, H. M. Rønnow, J. Mesot, O. Tjernberg, and J. Chang, Nodal Landau Fermi-liquid quasiparticles in overdoped $\mathrm{La}_{1.77} \mathrm{Sr}_{0.23} \mathrm{CuO}_{4}$, Phys. Rev. B 89, 205104 (2014).

[66] M. Horio, K. Hauser, Y. Sassa, Z. Mingazheva, D. Sutter, K. Kramer, A. Cook, E. Nocerino, O. K. Forslund, O. Tjernberg, M. Kobayashi, A. Chikina, N. B. M. Schröter, J. A. Krieger, T. Schmitt, V. N. Strocov, S. Pyon, T. Takayama, H. Takagi, O. J. Lipscombe et al., Three-Dimensional Fermi Surface of Overdoped La-Based Cuprates, Phys. Rev. Lett. 121, 077004 (2018).

[67] A. Narduzzo, G. Albert, M. M. J. French, N. Mangkorntong, M. Nohara, H. Takagi, and N. E. Hussey, Violation of the isotropic mean free path approximation for overdoped $\mathrm{La}_{2-x} \mathrm{Sr}_{x} \mathrm{CuO}_{4}$, Phys. Rev. B 77, 220502(R) (2008).

[68] Y. Fang, G. Grissonnanche, A. Legros, S. Verret, F. Laliberte, C. Collignon, A. Ataei, M. Dion, J. Zhou, D. Graf, M. J. Lawler, P. Goddard, L. Taillefer, and B. J. Ramshaw, Fermi surface transformation at the pseudogap critical point of a cuprate superconductor, arXiv:2004.01725.

[69] A. Yamamoto, W. Z. Hu, and S. Tajima, Thermoelectric power and resistivity of $\mathrm{HgBa}_{2} \mathrm{CuO}_{4+\delta}$ over a wide doping range, Phys. Rev. B 63, 024504 (2000).

[70] Z. Konstantinović, G. Le Bras, A. Forget, D. Colson, F. Jean, G. Collin, M. Ocio, and C. Ayache, Thermopower in the strongly overdoped region of single-layer $\mathrm{Bi}_{2} \mathrm{Sr}_{2} \mathrm{CuO}_{6+\delta}$ superconductor, Phys. Rev. B 66, 020503(R) (2002).

[71] T. Kondo, T. Takeuchi, U. Mizutani, T. Yokoya, S. Tsuda, and S. Shin, Contribution of electronic structure to thermoelectric 
power in $(\mathrm{Bi}, \mathrm{Pb})_{\cdot_{2}}(\mathrm{Sr}, \mathrm{La})_{{ }_{2}} \mathrm{CuO}_{6+\delta}$, Phys. Rev. B 72, 024533 (2005).

[72] D. G. Joshi, C. Li, G. Tarnopolsky, A. Georges, and S. Sachdev, Deconfined Critical Point in a Doped Random Quantum Heisenberg Magnet, Phys. Rev. X 10, 021033 (2020).

[73] P. Cha, N. Wentzell, O. Parcollet, A. Georges, and E.A. Kim, Linear resistivity and Sachdev-Ye-Kitaev (SYK) spin liquid behavior in a quantum critical metal with spin1/2 fermions, Proc. Natl. Acad. Sci. USA 117, 18341 (2020).
[74] H. Shackleton, A. Wietek, A. Georges, and S. Sachdev, Quantum Phase Transition at Nonzero Doping in a Random $t-J$ Model, Phys. Rev. Lett. 126, 136602 (2021).

[75] J. M. Park, Y. Cao, K. Watanabe, T. Taniguchi, and P. JarilloHerrero, Flavour Hund's coupling, Chern gaps and charge diffusivity in moiré graphene, Nature (London) 592, 43 (2021).

[76] B. Ghawri, P. S. Mahapatra, S. Mandal, A. Jayaraman, M. Garg, K. Watanabe, T. Taniguchi, H. R. Krishnamurthy, M. Jain, S. Banerjee, U. Chandni, and A. Ghosh, Excess entropy and breakdown of semiclassical description of thermoelectricity in twisted bilayer graphene close to half filling, arXiv:2004.12356. 